\title{
Integrated Si-Based Opto-Couplers: a Novel Approach to Galvanic Isolation
}

\author{
Antonio Alessandria, Luigi La Magna, Marco Renna, Leonardo Fragapane, Salvatore Coffa \\ STMicroelectronics Srl - Stradale Primosole, 50 - I - 95121 Catania - Italy \\ Antonio.ALESSANDRIA@st.com
}

\begin{abstract}
In many applications the need of galvanic isolation for safety or functional requirements is very high. In this paper a new isolated opto-coupler device concept is presented. Our approach combines an isolation technology with the integration of a silicon-based optical transmission system. This new concept enhances design, flexibility, performances and reliability of the devices, resulting in space and cost saving.

In the first section two isolation technologies will be presented. Subsequently, the transmission system will be elucidated. At last a case of application in the field of power devices will be presented.
\end{abstract}

\section{Introduction}

Nowadays many applications in different fields, ranging from industrial supply networks to data communications, require stringent features regarding safety and reliability. The way to guarantee the requirements is to provide galvanic isolation through a key-device; namely an insulator [1]. This device has to provide both electrical and signal isolation. This means that the insulator has to protect the surrounding circuitry and itself against physical damaging phenomena resulting from different voltage potential. For example we can examine the case of electrical and data network. Different nodes along the network may have different ground potential, and the potential variation between the nodes can result in unwanted current flow and ground loop, either AC or DC and possibly also with various noise components. Moreover the insulator must prevent the distortion of data through the rejection and filtration of common-mode transient.

Adding galvanic isolation to network circuits or components is often a good design practice, but is also frequently mandatory in industry standards. Most diffused isolation techniques employ discrete optocouplers, capacitive couplers, magnetic couplers or AC transformers. The main limitations in an isolator are linearity and frequency performance.

With our device concept we can obtain an isolator that overcomes some drawbacks of discrete optocouplers [2] and oversteps the performance of the existing isolators. The adoption of a silicon-based technology allows to benefit by the cost-effectiveness and renowned performance of silicon electronic circuits. Meanwhile it will open the way to monolithic integration of high-capacity, high value-added circuits (e.g. amplifiers, modulators, multiplexers, etc.) on the same isolator chip, and to fabrication of high-performance system-on-package (e.g. power devices with galvanic isolation, electrically-isolated data communication interfaces, etc.) [3].

The novel approach consists of a combination of a SOI isolation technique along with a silicon-based optical coupling system. In this paper we will explain our new device concept.

In the first section we will give some details about the insulation technique.

In the second section we will describe the parts of the optical transmission system; we will put some evidence on silicon-based light sources, waveguide and light detectors, operating at the wavelength of $1.54 \mu \mathrm{m}$.

In the third section we will present some cases of application of our device concept in the field of power devices. Then we will go through the conclusions.

\section{Galvanic isolation}

Galvanic isolation is a method to ensure no electrical connection between the input, output and ground terminals of a device.

Our device is a monolithic insulator build on a SOI substrate. In figure 1 the isolation technique is illustrated in a cross section.

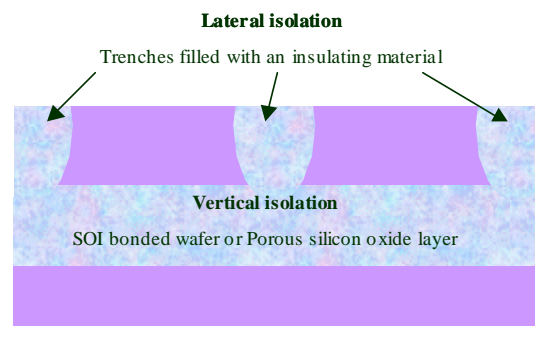

Figure 1. Isolation technique

Vertical isolation is obtained through a buried layer of $\mathrm{SiO}_{2}$. We are using two approaches to achieve vertical isolation:

- bonded SOI wafers;

- porous silicon oxide buried layer. 
While the first approach is standard [4], the second one represent an alternative low-cost solution. In this approach standard silicon wafers are prepared to form a buried porous silicon layer through a selective electrochemical etching process. Then the buried layer will be oxidized through a low-temperature, low-time oxidizing process. Figure 2 summarizes the process steps involved in the method.

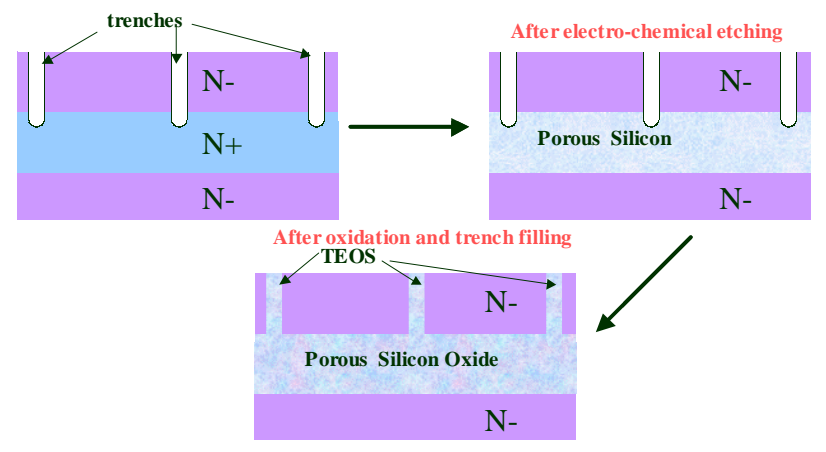

Figure 2. Insulating buried layer fabrication

It is important to underline that the oxidizing species can penetrate through the pores. This means that we have to oxidize only nano-crystalline dots remained in the structure. For this reason we can form thick oxide layer with small processes. Moreover the expansion of the dots due to different density between $\mathrm{Si}$ and $\mathrm{SiO}_{2}$, reduces the overall porosity of the layer after the oxidation. Further details about the process will be given in the full paper.

Lateral isolation is achieved through vertical trenches in the SOI layer reaching the insulating buried layer. Trenches are filled with an insulating material (CVD TEOS).

The total withstand voltage depends on many variables:

- buried oxide layer thickness;

- trenches width;

- dielectric constant of the filling material.

Moreover we have to face the problem of planarizing the structure, this means that we don't have unlimited freedom on the trenches width. In order to obtain a higher withstand voltage, a multi-trench method can be adopted (see figure 3). This method has also the advantage of bringing down any lateral capacitive coupling between the isolated regions.

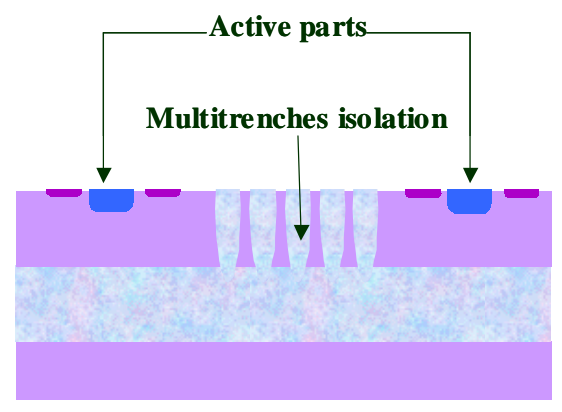

Figure 3. Multi-trench isolation method

\section{Optoelectronic devices integration}

In order to build a seamless path between input and output of the isolator, we need a system that allows a communication, different from the electrical one, between the isolated regions. In our device concept, communication is ensured through a silicon-based optoelectronic transmission system. In a transmission system like this we need:

- light sources;

- optical waveguides;

- light detectors.

Due to many reasons, which will be explained in the full paper, the selected wavelength is $1.54 \mu \mathrm{m}$.

In figure 4 our transmission system is illustrated.

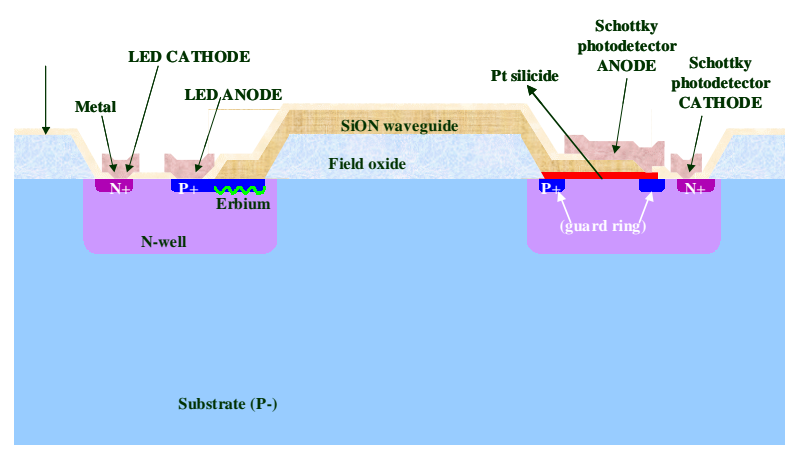

Figure 4. Optical transmission system schematic cross-section

We generate light emission through an Erbium-doped LED [5]. In this device radiative phenomena can arise from de-excitation of Erbium ions. Basically there are two mechanisms to excite an Erbium ion: electron-hole recombination and impact excitation from hot electrons. Non-radiative de-excitation of Erbium ions are very strong at room temperature and the best mechanism to produce light is to place Erbium ions in the depletion layer of a reverse-biased $p-n$ junction. Indeed in the depletion region the concentration of free carriers is very low and the non radiative mechanisms of de-excitation are inhibited.

Another way to generate light emission is to incorporate Erbium ions in the oxide layer of a MOS structure [6]. If we force current in the oxide of the MOS, electrons are accelerated with very high energy and impact excitation of Erbium ions is, consequently, very high. Moreover non-radiative excitation mechanisms of Erbium ions are not allowed in the oxide. MOS light sources have very high efficiency, but have strong reliability problems due to oxide deterioration.

Optimization of both, LED and MOS, light sources are in course. As regards the LED, we are trying to solve some integration problems, while regarding the MOS source, we are settling reliability issues with the introduction of a silicon-reach oxide layer in the MOS structure. Further details about integration and solutions will be given in the full paper. 
At the wavelength of $1.54 \mu \mathrm{m}$, Silicon is a good material to guide light. Unfortunately, we must remember that our device is an insulator, so Silicon is excluded from the selection of materials to fabricate waveguides. We need a material with appropriate refractive index and low absorption losses at $1.54 \mu \mathrm{m}$. Silicon oxynitride $\left(\mathrm{SiO}_{x} \mathrm{~N}_{y}\right.$ or $\mathrm{SiON}$ for short) seems to be a material with excellent optical properties and, moreover, its refractive index can be chosen in the range between 1.46 and 2 [7]. In our device the cladding of the waveguide is manufactured with VAPOX or thermal oxide $(\mathrm{n} \sim 1.46)$ while the core is fabricated with $\mathrm{SiON}$ with a refractive index of about 1.67. Some studies are in course to evaluate how SiON optical properties are modified due to integration (e.g. absorption losses vs thermal budget) and also to evaluate the coupling between optical components (LED or detector) and the waveguide (e.g. anisotrope wet etching to control the profile and the coupling angle).

Detection of light at the wavelength of $1.54 \mu \mathrm{m}$ in silicon is not simple. Indeed the maximum wavelength in the absorption window of silicon is about $1.13 \mu \mathrm{m}$. A way to obviate to this problem is to detect light at 1.54 $\mu \mathrm{m}$ with a Schottky diode. Whit such a diode photons with energy satisfying equation (1) can be detected.

$$
\phi_{B}<h v<E_{g}
$$

where $E_{g}$ is the Silicon bandgap, $v$ is the frequency and $\phi_{B}$ is the potential of the Schottky barrier. This means that our barrier must satisfy equation (2) to detect photons at $1.54 \mu \mathrm{m}$.

$$
\phi_{B}<8.055 E-1 \mathrm{eV}
$$

Some experiments are in course to manufacture Schottky barrier with Platinum silicide and to optimize the silicide thickness in order to reduce to the minimum the absorption in this layer. Other optimization are in course as regards topics related to the integration of the Schottky diode in the standard process flow used to manufacture electronic circuits. Additional information and results will be given in the full paper.

We want to focus that the main advantages of our device arise from integration. In few square millimeters of silicon we can integrate many transmission systems with very high density. Moreover we want to underline two characteristics of our device arising from integration:

- bi-directionality;

- multi-channels availability.

Further advantages and solutions about integration will be added in the full paper.

\section{Cases of application}

Optocouplers are adopted in many applications, but the presented new concept open the way to new frontiers. Our device concept allow to integrate in the same chip some electronic circuitry for special purpose (e.g. analog or digital processing of the input signals, amplification, modulation and demodulation, etc.). Moreover it is particularly interesting the assembly of different chips in the same package to offer a system-onpackage solution.

In this section we are going to present a power device for industrial application (e.g. an IGBT or a power MOS) with galvanic isolation. In figure 5 the schematic view of our system is depicted: chip A represents the integrated opto-coupler, including the driving circuitry for the power stage; chip B represents a monolithic smart power device. The two chips are assembled in the same double-island, single frame package. More information on the system-on-package solution will be presented in the full paper.

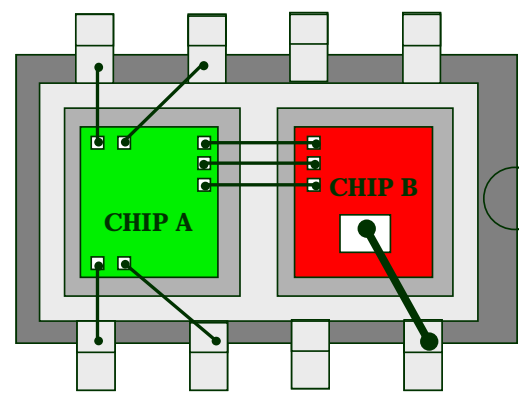

Figure 5. System-on-package schematic view

The presented device could be applied in different fields. An example could be the application of the device as solid state relée in a power meter. In this case the galvanic isolation is needed to ensure safety conditions for the users if bad operating conditions in the distribution lines occur.

A second example could be in the control circuit of a three-phase motor (see figure 6). In this case the device decouples the power stage (high voltages and high currents) from the low voltage stage (microcontroller).

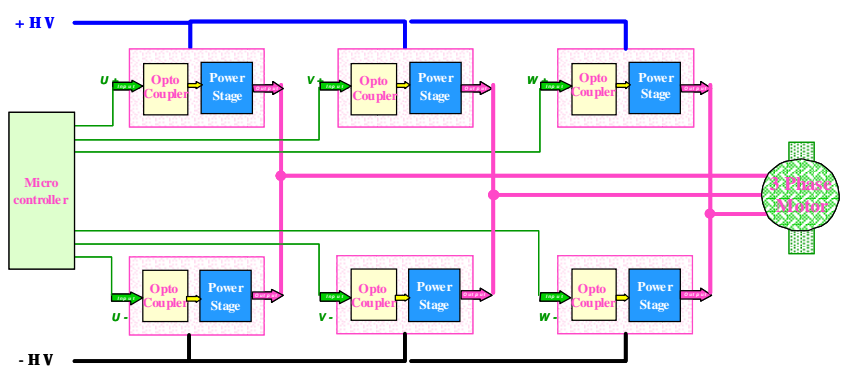

Figure 6. Motor control apllication

In the presented examples a safety isolation is required, that is the device has to withstand voltage over $2500 \mathrm{~V}$ and must have high values of common mode transient rejection. But there are many other applications in which is required only a functional isolation, as in the case of data communication interface. 


\section{Conclusions}

In the proposed paper a new concept to manufacture a galvanic isolator was presented. We adopt a monolithic solution to integrate some optoelectronic devices along with an isolation technology.

In the first part we have presented the isolation technology, then the transmission system was explained.

At last some examples of application were proposed.

Many experiments are in course and further details and results will be included in the full paper.

\section{References}

[1] T.M. Herman, W.C. Black, S. Hui, "Magnetically coupled linear isolator", IEEE Transaction on magnetics, Vol. 33, No. 5, September 1997, pp. 4029-4031

[2] J.B. Simoes, R.M.C. Silva, A.M.L.S. Morgado, C.M. Correia, "The optical coupling of analog signals", IEEE Transactions on nuclear science, Vol. 43, No. 3, June 1996, pp. 1672-1674
[3] U. Hilleringmann, K. Goser, "Optoelectronic system integration on silicon: waveguides, photodetectors, and VLSI CMOS circuits on one chip", IEEE Transactions on electron devices, Vol. 42, No. 5, May 1995, pp. 841-846

[4] B. Jalali, S. Yegnanarayanan, T. Yoon, T. Yoshimoto, I. Rendina, F. Coppinger, "Advances in silicon-on-insulator optoelectronics", IEEE Journal of selected topics in quantum electronics, Vol. 4, No. 6, November/December 1998, pp. 938947

[5] S. Coffa, S. Libertino, G. Coppola, A. Tutolo, "Feasibility analisys of laser action in Erbium-doped silicon waveguides", IEEE Journal of quantum electronics, Vol. 36, No. 10, October 2000, pp. 1206-1213

[6] S. Libertino, S. Coffa, M. Saggio, "Design and fabrication of integrated Si-based optoelectronic devices", Materials science in semiconductor processing, Vol. 3, 2000, pp. 375381

[7] R.M. De Ridder, K. Worhoff, A. Driessen, "Silicon oxynitride planar waveguiding structures for application in optical communication", IEEE Journal of selected topics in quantum electronics, Vol. 4, No. 6, November/December 1998, pp. 930-937 